\title{
The office based CHIVA
}

This article was published in the following Dove Press journal:

Journal of Vascular Diagnostics

25 September 2013

Number of times this article has been viewed

Fausto Passariello'

Stefano Ermini²

Massimo Cappelli ${ }^{3}$

Roberto Delfrate ${ }^{4}$

Claude Franceschi ${ }^{5}$

'Centro Diagnostico Aquarius, Napoli, Italy; ${ }^{2}$ Private Practice, Grassina, Italy; ${ }^{3}$ Private Practice, Firenze, Italy; ${ }^{4}$ Casa di Cure Figlie di Maria, Cremona, Italy; ${ }^{5}$ Hospital St Joseph, Service d'Explorations Vasculaires, Paris, France
Correspondence: Fausto Passariello

Centro Diagnostico Aquarius,

Via Francesco Cilea 280,

80127 , Napoli, Italy

Tel +3908I 7I 4 4II0

Email afunzionale@tiscalinet.it
Abstract: The cure Conservatrice Hémodynamique de l'Insuffisance Veineuse en Ambulatoire (CHIVA) can be office based (OB). The OB-CHIVA protocol is aimed at transferring CHIVA procedures to specialists rooms. The protocol will check the feasibility of OB-CHIVA, data pertaining to recurrence, and will offer the opportunity to study saphenous femoral junction (SFJ) stump evolution, the role of the washing vessels and the arch recanalization rate, and gather new data about the effect of the length of the treated saphenous vein. A simplified diagnostic procedure will allow an essential ultrasound examination of the venous net while a schematic and easily readable algorithm guides therapeutic choices. The Riobamba draining crossotomy (RDC) tactic is composed of a set of OB procedures. While some of these procedures are, at the moment, only proposals, others are already applied. Devices generally used in ablative procedures such as Light Amplification by Stimulated Emission of Radiation (LASER), radio frequency, steam, and mechanical devices are used in this context to serve to conservative interventions for CHIVA. New techniques have also been proposed for devalvulation and tributary disconnection. Detailed follow-up is necessary in order to determine the effects of therapy and possible disease evolution. Finally, information is added about the informed consent and the ethical considerations of OB-CHIVA research.

Keywords: CHIVA, office based procedures, LASER, RF, steam

\section{History and anecdotal cases}

The Riobamba laser draining crossotomy (RLDC) was designed in 2009 by Passariello in the city of Riobamba, Ecuador. Some cases were performed following the present protocol on Shunt I and I+II configurations, in collaboration with the surgical team of Morrison and later of King.

The cure Conservatrice Hémodynamique de l'Insuffisance Veineuse en Ambulatoire (CHIVA) can be office based (OB). The need for an OB-CHIVA intervention arose because only simplified resources were available in a non-typical surgical setting. The OB-CHIVA was introduced in order to reduce the required resources and generic surgical risk, especially risks associated with CHIVA crossotomy. In addition, OB-CHIVA tries to answer the "often faced but never solved issue" of a minimally invasive surgical CHIVA intervention.

The objective of OB-CHIVA is to ensure that it is quick and easy (or hopefully a little bit quicker and easier) than the currently available $\mathrm{OB}$ ablative procedures. $\mathrm{OB}-$ CHIVA was presented to the II Hemodynamic Conference in Phoenix in $2009,{ }^{1}$ to the XXIII Annual Congress of the American College of Phlebology Meeting in Palm Desert, California also in $2009,{ }^{2}$ and to the XI Meeting of the Association Europea de Chiva (AECH) in Puerto Madryn, Argentina in 2010;,3 where the OB-CHIVA issue was also chosen as a topic for the next meeting in Hannover. 
The actual version of the protocol is essentially a thorough detailed extension that allows the treatment of other network configurations. In addition, a modified format will accept also cases made using different techniques.

The protocol requires a minimal basic knowledge of ultrasound venous examination. In addition, non-detailed knowledge of hemodynamic surgery CHIVA is preferred. However, everyone should be able to read the text, as all required information is available in the protocol.

\section{CHIVA and OB-CHIVA}

\section{Premise}

CHIVA is a hemodynamic strategy, which requires an adequate tactic. The procedures of the OB-CHIVA here described to fit the tactic to the office environment could show in this experimentation inadequate results, compared to classic CHIVA interventions. It is better to declare a substantial difference between CHIVA and OB-CHIVA. The latter is not only a technical but rather a conceptual variation of CHIVA, thus they cannot be considered the same thing.

\section{CHIVA}

Acronym

CHIVA is an acronym for cure Conservatrice Hémodynamique de l'Insuffisance Veineuse en Ambulatoire (Conservative and Hemodynamic treatment of Venous Insufficiency in Outpatients).

\section{Strategy}

CHIVA is a 'strategy' of treatment of venous diseases applied to outpatients and based on hemodynamic concepts, which aims:

- to fractionate the hydrostatic pressure

- to disconnect the venovenous shunts

- to preserve the compensating and draining veins, even if varicose.

Strategy can improve/evolve with the progress of knowledge in venous pathophysiology and diagnosis.

\section{Tactics}

CHIVA is also a tactics composed of tools used to achieve the purpose of the strategy.

Tactics can improve with the techniques of venous disconnections. So far, open surgery is the best tactics (local anesthesia and mini-invasive surgery). Saphenous femoral junction (SFJ) and saphenous popliteal junction (SPJ) CHIVA crossotomy is performed only in part of the hemodynamic configurations, is mini-invasive, and safe when echo-guided. Currently available choices for CHIVA crossotomy are section ligature, isolated ligature, and the clip and the triple saphenous flush ligation (TSFL) technique.

Endovenous procedures will be welcome when they will comply to the CHIVA strategy and will achieve long lasting disconnections.

\section{OB-CHIVA}

\section{Acronym}

OB-CHIVA is an acronym for Office Based-CHIVA.

\section{Strategy}

OB-CHIVA is a slightly different strategy, which attempts to transfer CHIVA treatment from the operating room to the specialist room. This environment change requires some adjustments in CHIVA strategy. Synthetically, strategy is modified because the disconnection is placed below some arch tributaries (not a flush disconnection) and a limited part of the greater saphenous vein (GSV) is destroyed. Differences are listed in Table 1.

\section{Tactics}

OB-CHIVA is also a tactics composed of a set of therapeutic procedures, freely chosen by the operator between adapted endovenous procedures like Light Amplification by Stimulated Emission of Radiation (LASER), RF, steam, and mechanical ablation. The choice is free because at the moment there is no demonstration of the superiority of any one of them. Other complementary procedures include several methods of devalvulation and the tributary access.

Table I Detailed differences between CHIVA and OB-CHIVA

\begin{tabular}{lll}
\hline & CHIVA & OB-CHIVA \\
\hline Washing & $\begin{array}{l}\text { None in GSV, } \\
\text { sometimes used } \\
\text { in SSV }\end{array}$ & Always \\
& $\begin{array}{l}\text { All the arch } \\
\text { tributaries } \\
\text { (no one is ligated) }\end{array}$ & $\begin{array}{l}\text { Some tributaries downward } \\
\text { the treated arch segment }\end{array}$ \\
& $\begin{array}{l}\text { Absent } \\
\text { (flush ligation) }\end{array}$ & $\begin{array}{l}\text { Small stump with washing } \\
\text { vessels }\end{array}$ \\
Stump & Only SFJ & Generally the far group of \\
incompetent & SFJ tributaries or all the ones \\
tributaries & tributaries & lower than an incompetent \\
Invasivity & Surgical & one, if any \\
Environment & Ambulatorial & Endovenous \\
\hline
\end{tabular}

Abbreviations: CHIVA, cure Conservatrice Hémodynamique de I'Insuffisance Veineuse en Ambulatoire; GSV, greater saphenous vein; OB, office based, SFJ, saphenous femoral junction; SSV, shorter saphenous vein. 
Tactics could improve, and couldn't be limited only to adapted endovenous procedures.

The great challenge in the future is to find an alternative office based procedure which complies with the CHIVA strategy, thus transforming OB-CHIVA into a tactical variation of CHIVA.

Nowadays however OB-CHIVA shouldn't be confused with CHIVA, as in strategy as in tactics. Also the argument that it is less invasive than surgery is disputable, as a study comparing classic CHIVA versus classic ablative LASER has shown that CHIVA is less painful. ${ }^{10}$

\section{OB-CHIVA protocol}

Anecdotal cases of OB-CHIVA were performed since from the start (Riobamba, 2009) gathering data in a systematic, but unwritten way.

The OB-CHIVA protocol is an attempt to provide a welldefined guideline to the adopted procedures in OB-CHIVA. Also diagnosis is specified in details in order to avoid elementary and trivial errors. As an example, several configuration hints are given in order to adequately set the ultrasound devices.

The protocol aims also to make OB-CHIVA simpler, especially in diagnosis, where the use of a flow chart can simplify, in a sensible way, the comprehension of hemodynamics. The use of complex terms about the shunt classification are carefully avoided, in order to capture a wider audience and to stimulate operators generally never involved in CHIVA before.

The OB-CHIVA protocol is actually used in research, though in the future it could be used in a simplified way, in daily clinical work. Research results will hopefully give hints about how to simplify procedures, and reduce requirements and costs. However, at the moment, searching for timesaving shortcuts isn't the main aim and the protocol must be as comprehensive as possible to include current, as well future, methods.

\section{Detailed differences}

Some arch tributaries are sacrificed in OB-CHIVA so, in theory, a difference could exist in drainage and varicose veins recurrence rates.

Tributaries of the GSV arch have great variability, but generally it is possible to divide them in two groups (Table 1):

- The near to SFJ group is composed of the epigastric, pudendae, and iliac circumflex tributaries (often a common trunk with the epigastric).

- The far from SFJ group. Apart from the near incompetent tributaries (which must always be sacrificed), only the far group is a candidate to be eliminated using the endovenous procedure.
Using washing vessels leaves a small stump at the SFJ. The use of washing vessels was introduced for the shorter saphenous vein (SSV) CHIVA treatment, ${ }^{5,6}$ but became a standard in SFJ thermal ablation, and is practiced almost everywhere. However, no reliable study is available on this issue.

\section{Analogies}

LASER closure can only be regarded as a different tactical method to eliminate SFJ reflux with a CHIVA crossotomy (Figure 1A-C). Other currently available methods to perform CHIVA crossotomy are section-ligature, isolated ligature, the clip, and triple saphenous flush ligation (TSFL) technique; in the same way it occurs with all these methods, also in OB-CHIVA a recanalization is possible. ${ }^{7}$

\section{Aim}

In this preliminary open study the aim is:

- To check using greater numbers, the feasibility of the OB-CHIVA, by reviewing more than the first anecdotal results.

- To set a comparison among detected varicose veins recurrence rates and data taken from the literature regarding CHIVA recurrence.

- To gather data regarding SFJ stump evolution and the role of washing vessels.

- To determine the arch recanalization rate of the procedure.

\section{Ultrasound device settings}

\section{Steering}

None; a rectangular box is used.

\section{Direction}

Set a positive signal (by convention red) for the towardprobe flow and a negative signal (by convention blue) for the away-probe flow.

\section{Range}

The same range is used for pulsed wave (PW) and color Doppler. In order to get good sensitivity at slow velocities, set a $\pm 5 \mathrm{~cm} / \mathrm{s}$ or $\pm 10 \mathrm{~cm} / \mathrm{s}$ interval, while higher values will either fall at the limit of the aliasing or over it.

\section{GSV flow detection}

\section{General rules}

Transverse cross-section: the probe is directed upward, at 45 degrees to the skin. The probe detects the velocity component directed toward the probe, positioned forming an angle of 45 degrees with the vessel. A positive signal is 
A
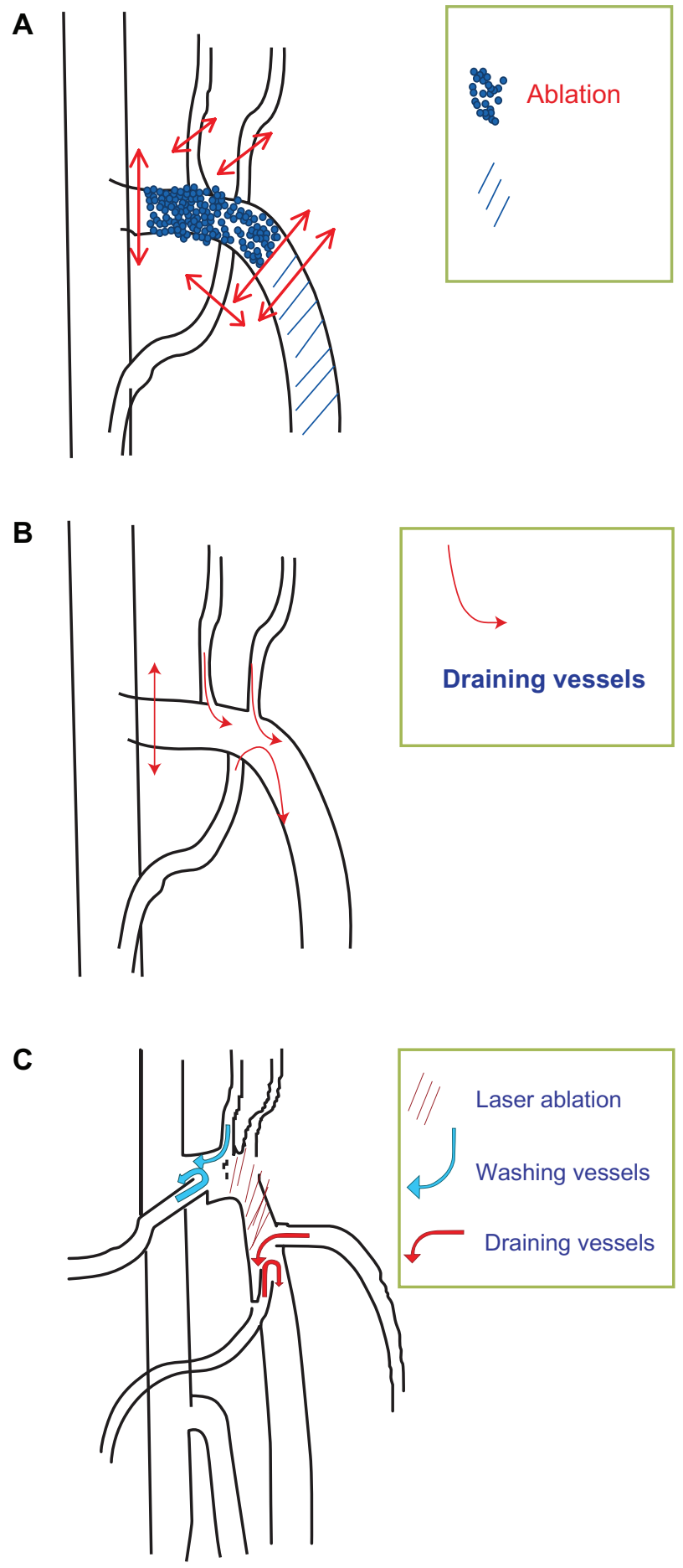

Figure I Differences between several methods for treating the saphenous arch: (A) traditional crossectomy; (B) CHIVA crossotomy; (C) RLDC Crossotomy (LASER). Abbreviations: CHIVA, Cure Conservatrice Hémodynamique de l'Insuffisance Veineuse en Ambulatoire; LASER, light amplification by stimulated emission of radiation; RLDC, Riobamba laser draining crossotomy.

a descending/refluxing flow, while a negative signal is an ascending/physiologic flow.

\section{GSV trunk}

The angle line must be absent or vertical.

\section{SFJ terminal valve}

The longitudinal section requires no steering: the screen must visualize the common femoral vein (CFV) and the SFJ. This can be accomplished by putting the sample volume in the CFV just near the SFJ. The angle line must be directed into the SFJ, as we want to detect SFJ incompetence. Aligning instead with the CFV direction detects only CFV incompetence. A positive signal is a toward the surface/refluxing flow, a negative signal is a centripetal/ physiologic flow.

Transverse cross-section is able to visualize the commissural reflux. The probe must visualize the CFV and the SFJ (two near circles). This can be accomplished by putting the sample volume in the CFV just near the SFJ. The angle line must be directed into the SFJ.

\section{SFJ pre-terminal valve}

The same section is used as for terminal valve. No steering is required; the sample volume is put on the arch, immediately after the SFJ. In the longitudinal section, the angle line must be aligned with the SFJ arch. A positive signal is a toward the surface/refluxing flow, a negative signal is a centripetal/ physiologic flow.

\section{Reflux detection}

Use the Valsalva maneuver elicited by insufflating (systole) into a common drinking straw closed at one terminal with a knot (Franceschi straw, Figure 2). In a valid maneuver, flow must revert to the physiologic direction during rebreathing (diastole).

\section{SFJ anatomy}

Count the number of tributaries to be used as washing vessels (\#wash) and measure the max washing caliber ( $\phi$ wash), and the distance from the SFJ to the last arch washing tributary (SFJ-free in $\mathrm{cm}$ ).

Count the number of tributaries to be used as draining vessels (\#drain) and measure the max draining caliber ( $\phi$ drain), and the GSV length (L) to be treated (in $\mathrm{cm}$ ) from the last washing to the first draining tributary. Draining and washing vessels are defined as part of the project before the intervention. During the intervention, the surgeon tries to respect the project.

During the follow-up, the ultrasonographer detects if the draining and washing vessels are still open, their number, and their caliber.

\section{Protocol design}

The protocol includes only OB-CHIVA cases with crossotomy performed with alternative methods (LASER, RF, 


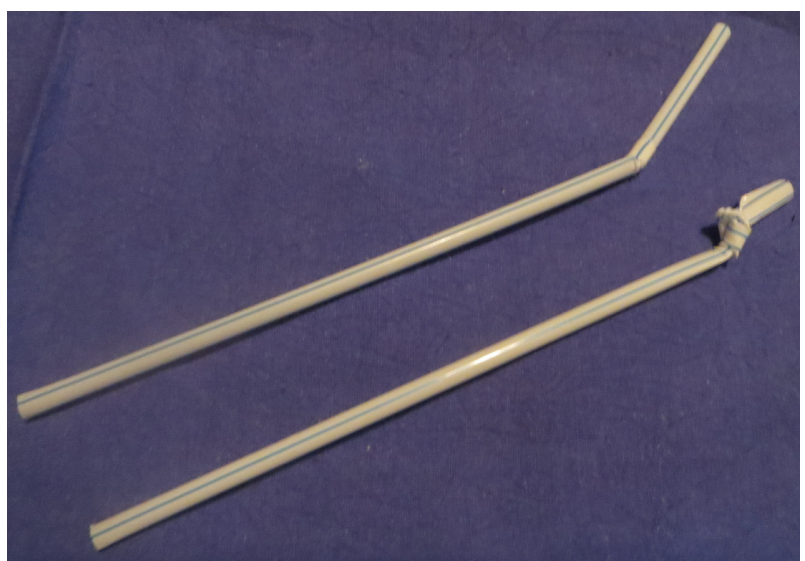

Figure 2 A drinking straw (top) and a Franceschi straw (make a knot) (bottom).

and steam). Chemical agents were excluded because at the moment they are not able to get localized and limited extent effects on veins.

\section{Exclusion criteria}

Exclusion criteria were: deep veins actual involvement (thrombosis or incompetence); previous SVT; pelvic shunt (a Valsalva increase in descending flow in the upto-down branches of the GSV arch); and commissural SFJ reflux.

\section{Inclusion criteria}

A correct indication can be achieved synthetically using shunt definitions. However, the same result is obtained by avoiding the hemodynamic terms as follows:

The prerequisite for treatment is SFJ TV incompetence. If this is the case, in order to classify and understand the hemodynamic behavior of the system we need to know: if the GSV reflux is deviated or not (Shunt I) towards at least one incompetent tributary; and if the latter is the case, does the GSV trunk reflux disappear? (positive reflux elimination test [RET]+, Shunt III) or not ? (negative reflux elimination test [RET]-, Shunt I+II) while pressing the tributaries with a finger (with a little help from our collaborators, as sometimes you need more than two hands to perform the test). ${ }^{8}$

This evaluation grid permits the classification and choice of the therapy (Figure 3).

\section{Number of cases}

This is an open study; there is no control on the number of cases. The protocol will end when at least one of the two following conditions will be satisfied: 500 treated cases or 2-years from the official publication. Follow-up on last

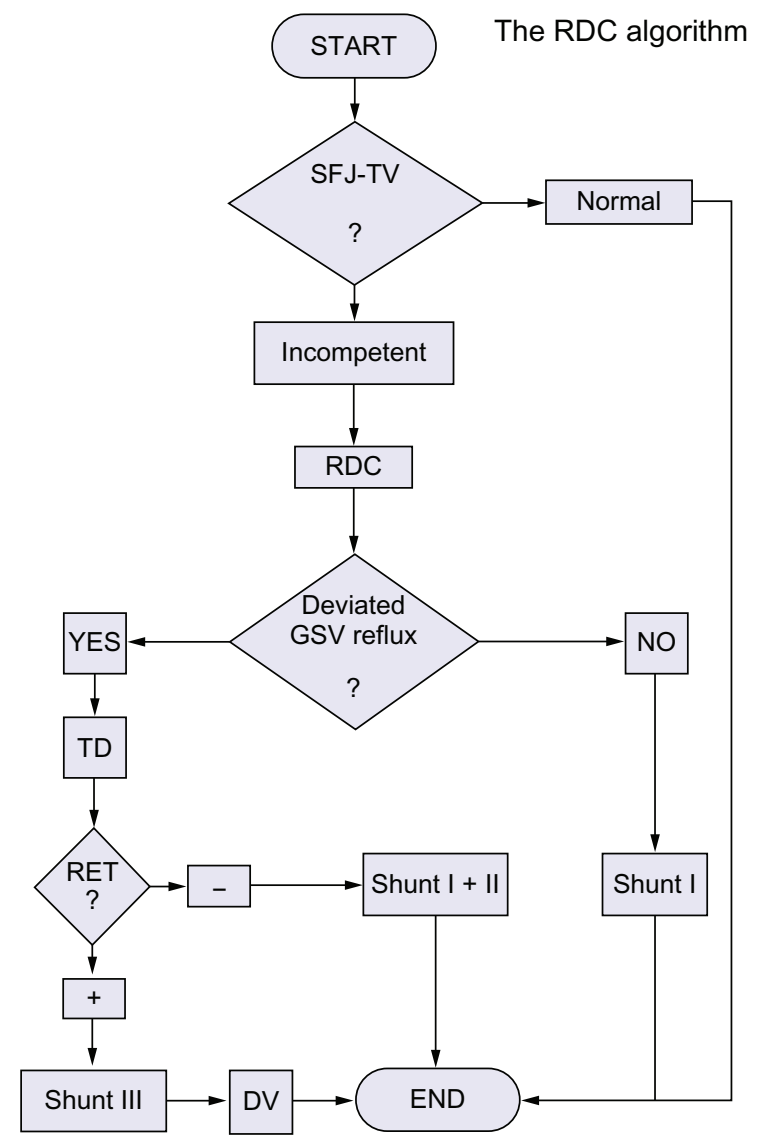

Figure 3 The flow chart of the RDC algorithm.

Abbreviation: RDC, Riobamba draining crossotomy; SFJ, saphenous femoral junction; TV, terminal valve; TD, tributary disconnection; RET, reflux elimination test; +, positive; -, negative; DV, devalvulation.

treated cases could however go on to gather more data. The analysis of the results will then be a guide for setting up a more robust protocol (with the same or a modified method) or will suggest abandoning the study.

For each patient the individual height, the height of the groin and of the re-entry perforator, the venous clinical severity score (VCSS) ${ }^{11}$ and for quality of life (QoL), the Aberdeen varicose veins questionnaire (AVVQ) ${ }^{12}$ and the Research AND Development (RAND)-36 ${ }^{13}$ will all be reported.

\section{Riobamba draining crossotomy tactics \\ Riobamba laser draining crossotomy}

For RLDC, ${ }^{9}$ the tip of the LASER fiber is placed at the end of the last washing tributary, at a distance from the junction worth SFJ-free cm and one L length segment is treated until the first draining tributary.

The Power (w) and the pullback speed of the fiber (v) are recorded and the treated length (LT), which generally differs from L, is measured. Finally, the administered Energy (E) 
and the linear endovenous energy density (LEED) = power/ velocity $=\mathrm{w} / \mathrm{v}$ are computed or, even better, measured.

For a typical therapy $\mathrm{w}=14 \mathrm{~W}, \mathrm{v}=0.1 \mathrm{~cm} / \mathrm{s}$, and LEED $=$ $140 \mathrm{~J} / \mathrm{cm}$.

Also, the use or lack of use of a catheter and of the tumescence, with the temperature, composition, and volume of the components (saline or bicarbonate; including the percentage), and adopted anesthetics should all be reported.

\section{Fixed RLDC}

Starting as described after the last washing vessel, a fixed length of the trunk is treated (see the RRDC paragraph below). This simplification in the procedure does not authorize the omission of a detailed study of the SFJ, as the measurements will make it possible to understand if the fixed length method can really be compared to the ultrasound guided detailed study of the GSV arch.

\section{Riobamba radio frequency draining crossotomy}

Riobamba radio frequency draining crossotomy (RRDC) is a very precise procedure with a fixed length treatment. RF works in cycles of 20 seconds, reaching a target temperature of $120^{\circ} \mathrm{C}$. During catheter pullback, the device automatically regulates the emitted power to maintain the temperature. Planning the therapy requires only choosing the number of cycles per segment and the number of segments.

The electrode at the tip of the catheter is $7 \mathrm{~cm}$ long, though a new available electrode provides a length of $3 \mathrm{~cm}$. Sequential electrode positions can usually overlap for $0.5 \mathrm{~cm}$ and the treated segments are almost 6.5 or $2.5 \mathrm{~cm}$ long.

In OB-CHIVA, a few segments near the SFJ are treated:

- for a length of $7 \mathrm{~cm}$ (no overlap) or $13.5 \mathrm{~cm}$ ( 2 overlapped segments) using the greater $7 \mathrm{~cm}$ electrode

- for a length of $3 \mathrm{~cm}$ (no overlap) or $5.5,8.0,10.5 \mathrm{~cm}$ (respectively 2, 3, 4 overlapped segments) using the smaller $3 \mathrm{~cm}$ electrode.

Thus the only variables to be fixed are the number of cycles (the overall time length per segment) and the number of segments.

Though RRDC is a fixed length procedure, the choice of the smaller electrode could fit a detailed length to be treated.

\section{Riobamba steam draining crossotomy}

The use of steam is only a fascinating hypothesis. As the steam jet effect decreases with distance, it is only possible to use a fixed length from the SFJ-free point to treat a GSV segment. For this reason, Riobamba steam draining crossotomy (RSDC) could only be a fixed length procedure.

\section{Riobamba mechanical draining crossotomy}

Riobamba mechanical draining crossotomy (RMDC) is a good candidate to compete with LASER for its precision.

\section{Wire guided devalvulation}

You can make a classic devalvulation during a flush phlebectomy or a wire guided devalvulation (WGD), using a 18 gauge needle, a wire guide, and a dilatator, generally included in the catheter kit.

\section{WGD virtual dissection}

If the distance from the GSV access point to the valve is less than the dilatator length, a WGD virtual dissection can be used. Using the same GSV venous access as SFJ treatment, retract the needle until only the tip is inside the vein, then pull the skin upwards, invert the needle direction, and push it into the vein and then insert the wire guide. Next, retract the needle completely and stop if needed, releasing the skin. At this moment, the wire inside the vein is in the inverted direction. Next, pull the skin upward and insert the dilatator until you reach the valve. Don't release the skin and compress upward to close the valve. Now, push the dilatator and pass through the valve many times. Finally, retract the dilatator and release the skin. The entire procedure should be followed by US, checking the phases in the sequence.

If a catheter is used as a fiber sheath, the start of the procedure is different: remove the fiber (unlock it) and reinsert the wire before completely removing the sheath. Now, insert the needle following the wire until the tip enters the vein. Remove the wire. The remaining part of the procedure is identical as previously mentioned.

\section{Tributary disconnection}

Disconnection can be performed classically by a flush phlebectomy, which is an ambulatory/office quick procedure. Also, LASER and foam can be used with different effects.

\section{Tributary access}

The tributary venous access (proposed by Ermini [personal communication, 2013]) could be used as an alternate way to reach and treat the SFJ and it can be used with any device. 
Performing the tumescence when needed, treating and then retracting the device allows a passage through the tributary just before exiting. A new peripheral tumescence and treatment allows then a single access double procedure, which reduces execution time and invasivity. This method cannot be applied when the reflux is not deviated into an incompetent tributary, though these cases are rare.

In case of a deviated reflux with a positive reflux elimination test a devalvulation strategy could be chosen. However, the tributary access method does not allow the reuse of the same venous access through a wire guided virtual dissection; thus, the GSV is accessed directly in the downward direction.

\section{Follow-up}

A follow-up examination will be performed soon after the procedure at 1-week, 1-month, 6 months, 1-year, and 2-years.

For each follow-up examination, the GSV reflux, the tributary reflux, SFJ free, \#wash, фwash, \#drain, and the $\phi$ drain should be recorded.

In addition, the length of the GSV segments of total and partial occlusion above and below the treated zone should be measured and the state of the phlebectomies should be reported.

Pigmentation and any thrombosis in extra-saphenian segments should be detected and any notes considered important as well as the VCSS, the AVVQ, and RAND-36 scores should be reported.

\section{Informed consent}

Informed consent consists of a general part common to other procedures cited in the protocol and a specific one, which deals with the choice of the procedure. Essentially, the patient should be aware of the advantages and disadvantages of classic CHIVA surgery, the proposed OB-CHIVA, and the other procedures, including ablative (foam) and conservative (ASVAL). Another issue involves the type of OB-CHIVA procedure to be applied. Generally, as the OB-CHIVA protocol is open also to small centers where only a limited number of procedures are available, it can be assumed that only a few methods are proposed, although the information provided in the informed consent must be complete.

Essentially CHIVA and OB-CHIVA are conceptually different, though they share in part the same strategy. However, they differ strongly in the tactical choices. Thus, adequate information should be provided in order for the patient to make an educated choice.
The patient should also be informed about the loss or preservation of the saphenous heritage, its potential use in cardiac or arterial diseases, and the availability and cost of new materials for arterial grafts.

\section{Ethical considerations}

Though some randomized control trials ${ }^{10}$ comparing stripping and CHIVA, LASER and CHIVA, and so on, are available at the moment, no clear recognized superiority is assigned to any one procedure.

OB-CHIVA is a slightly modified CHIVA strategy designed to use some technical facilities adopted in ablative procedures. The essential difference is the reduced length of saphenous treatment, which is much shorter than the length generally treated in laser procedures $(25-35 \mathrm{~cm})$.

However, no reliable study is available about the length of treatment of the saphenous trunk. Thus, this study does not oblige an irrational technical choice and instead could help clarify the effect of the venous length on the treatment.

Unlike CHIVA but similar to LASER and RF, OBCHIVA leaves some tributaries of the arch and uses them as washing vessels. This cannot constitute an ethical issue as it is a technical choice commonly practiced in several procedures. Thus, the protocol is ethically acceptable and could serve to study the evolution of SFJ stumps.

These ethical considerations should be submitted to an Ethical Committee, if needed in the health structure where the protocol is applied, whether in a public, accredited, or partially/totally private institution.

Local changes are required to comply with local laws and common uses. A translation of the current text could be required, according to local laws.

Finally, essential help should be given locally by centers which join the protocol, as they know better, the local laws.

\section{No disclosure agreement}

During the experiment, this research will have a public content, ie, published in a scientific magazine and on a website; and a private content, ie, disclosed only to joining centers and supporting companies, consisting of details, forms, and organization texts, and partial results and conclusions, as well as partial changes to the procedures to assure better management of the research.

People, centers, and companies joining the protocol will be requested to sign a non-disclosure agreement in order to avoid the diffusion of private contents. 


\section{Disclosure}

The authors report no conflicts of interest in this work.

\section{References}

1. Passariello F. The II Hemodynamic Conference. American Foundation of Phlebology, Phoenix (AZ, USA), November 2-3, 2009.

2. Passariello F. Venous Hemodynamics. Invited presentation to the American College of Phlebology Meeting, Palm Desert (CA, USA), November 5-8, 2009.

3. Passariello F. Office Based (OB) C.H.I.V.A. Presented to the XI Meeting of the AECH, Puerto Madryn, Argentina, Oct 7-8, 2010.

4. Passariello F. Office Based Chiva (OB Chiva). Acta Phlebol. 2011;12: 26-27.

5. Passariello F. Nuove strategie per la Chirurgia della Safena esterna. In: Passariello F. La Chirurgia della Safena Esterna. Napoli: New Print; 1991.

6. Passariello F, Carbone R. Chirurgia dell' Arco della Safena Esterna. (SSV Arch Surgery) Min. Angiol. vol. 1992;17(2 Suppl 3);149-156. Ed. Min. Medica, Torino (Aprile-Giugno 1992).
7. Delfrate R. Manuale di Emodinamica venosa degli arti inferiori. [A Primer of Venous Hemodynamics of Lower Limbs]. Cremona, Italy. Edizioni Fantigrafica, 2010. Italian.

8. Franceschi C, Zamboni P. Principles of venous hemodynamics. 2009; Nova Science Publisher Inc; 2009.

9. Passariello F, Hogue R, Goldman MP, et al. The WHAT-MD classification for the venous interventions. The pre-release preliminary version 4. Acta Phlebol. 2011;12:21-25.

10. Chan CY, Chen TC, Hsieh YK, Huang JH. Retrospective comparison of clinical outcomes between endovenous laser and saphenous vein-sparing surgery for treatment of varicose veins. World J Surg. 2011;35(7):1679-1686.

11. Vasquez MA, Munschauer CE. Venous clinical severity score and quality-of-life assessment tools: application to vein practice. Phlebology. 2008;23:259-275.

12. Ward A, Abisi S, Braithwaite BD. An Online Patient Completed Aberdeen Varicose Vein Questionnaire Can Help to Guide Primary Care Referrals. Eur J Vasc Endovasc Surg. 2013;45:178-182.

13. Hays RD, Sherbourne CD, Mazel RM. The RAND 36-item Health Survey 1.0. Health Econ.1993;2:217-227.
Journal of Vascular Diagnostics

\section{Publish your work in this journal}

Journal of Vascular Diagnostics is an international, peer-reviewed journal of diagnostics, focusing on non invasive vascular investigation methods involved in the evaluation of vascular diseases. The journal is committed to the rapid publication in the fields of vascular diseases. Original research, review, case reports, expert opinion and commentaries

\section{Dovepress}

are all considered for publication. The manuscript management system is completely online and includes a very quick and fair peer-review system, which is all easy to use. Visit http://www.dovepress.com/ testimonials.php to read real quotes from published authors. 